\title{
Bayesian Detection of Radar Interference in
}

\section{Radio Astronomy}

\author{
Brian D. Jeffs, Weizhen Lazarte, \\ Department of Electrical and Computer Engineering, Brigham Young University, Provo, UT 84606; \\ (bjeffs@ee.byu.edu,wzhdong@hotmail.com) \\ and J. Richard Fisher \\ National Radio Astronomy Observatory, 520 Edgemont Road, Charlottesville, VA 22903-2475, USA; \\ (rfisher@nrao.edu)
}

\begin{abstract}
L-Band observations at the Green Bank Telescope (GBT) and other radio observatories are often made in frequency bands allocated to aviation pulsed radar transmissions. It is possible to mitigate radar contamination of the astronomical signal by time blanking data containing these pulses. However, even when strong direct path pulses and nearby fixed clutter echoes are removed there are still undetected weaker aircraft echoes present which can corrupt the data. In a previous paper we presented an algorithm to improve real-time echo blanking by forming a Kalman filter tracker to follow the path of a sequence of echoes observed on successive radar antenna sweeps. The tracker builds a history which can be used to predict the location of upcoming echoes. We now present details of a new Bayesian detection algorithm which uses this prediction information to enable more sensitive weak pulse acquisition. The developed track information is used to form a spatial prior probability distribution for the presence of the next echoes. Regions with higher probability are processed with a lower detection threshold to pull out low level pulses without increasing the overall probability of false alarm detection. The ultimate result is more complete removal, by blanking the detected pulse, of radar corruption in astronomical observations.
\end{abstract}

\section{Index Terms}

RFI mitigation, radar RFI mitigation, Kalman tracking, Bayesian detection.

\section{INTRODUCTION}

Radio astronomers must often share spectrum with other licensed radio services when observing deep space objects. For example, though the neutral Hydrogen emission line at 1,420.4 MHz lies in a protected band, moderate red shifting seen in distant sources can move the signal into the 1,230-1,350 $\mathrm{MHz}$ range where many high power air surveillance radar transmitters operate. These impulsive sources are ever present. Even when the telescope is

This work was supported by the National Science Foundation grant number AST-9987339 
located in a radio quiet zone surrounded by mountains blocking the direct line of sight signal path, echoes from aircraft hundreds of kilometers away can corrupt sensitive observations [1], [2], [3].

This paper reports on efforts to mitigate radar interference at the NRAO Green Bank observatory in West Virginia. Pulses from the ARSR-3 surveillance radar located $104 \mathrm{~km}$ to the south southeast have corrupted Green Bank Telescope (GBT) observations at 1,292 MHz even when signals arrive in the telescope deep sidelobes. Since radar transmissions are impulsive with a relatively low duty cycle, the customary mitigation approach is to "blank" or excise data samples containing corrupting echo pulses. The remaining data is still suitable for long integration power spectrum analysis. The challenge lies in detecting interfering pulses so that they can be removed.

In a previous companion paper [4] we proposed a Kalman tracker to follow a sequence of aircraft echo detections seen at a radio telescope. The track history was used to guide real-time predictive blanking. It was also suggested briefly that track information could be used to improve detection performance. In this present paper we further develop that concept, present a detection theoretic basis for a new Bayesian detection method, and analyze detection performance improvement using Monte Carlo simulation. We refer the reader to [4] for a more detailed description of the interfering radar signal characteristics and Kalman tracker implementation.

The principal advantage of the proposed Bayesian method is that it enables detection of weaker echoes than is possible using conventional methods. This can improve track quality by reducing the number of missed detections arising from normal random fluctuations in aircraft radar cross section (and thus echo intensity). Furthermore, since in radio astronomy we are interested in signal levels below the noise floor, weak undetected echoes can still have higher instantaneous power levels than the signal of interest, and can thus bias observations. Any method that improves weak echo detection can potentially reduce the amount of corrupted, unblanked data used in signal analysis.

In the proposed detection algorithm, Kalman track histories consisting of a series of detected echoes from each aircraft are used to form a spatially dependent prior probability model for echo arrival. This prior distribution enables the Bayesian detector approach which increases probability of echo detection (PD) without increasing the effective probability of false alarm (PFA, i.e. the probability of declaring an echo detection when none is present).

The sampled radio telescope signal is first processed by a conventional radar digital matched filter receiver to produce a complex baseband time series, $b[n]$. Details for the radar receiver design are found in [5] and [4]. Receiver output $b[n]$ is reordered for detection processing to form a 2-D range-azimuth map $b(R, \theta)$ as shown in Figure 1. Each column of pixels contains receiver output samples from one transmitter pulse interval. Each time sample $n$ maps to a corresponding point in space $p$ located at the center of a range-azimuth bin (or pixel). The midpoint of bin $p$ has Cartesian coordinates $\left(x_{p}, y_{p}\right)$ and polar coordinates $\left(R_{p}, \theta_{p}\right)$. In expressions where geometrical position is more meaningful than time sample index, $b_{p}$ will be used interchangeably with $b[n]$, and serves as a shorthand notation for bin $p$ in the range-azimuth map, $b\left(R_{p}, \theta_{p}\right)$.

Detection processor outputs are used both to guide blanking and as inputs to the Kalman tracker (see [4]). The tracker creates detection histories and predicts locations for the next anticipated echoes. These prediction points are utilized in turn by the Bayesian detector as prior probability inputs to adjust detection sensitivity as a function of 
spatial position.

Sections II and III present the underlying probability models and theoretical algorithm development respectively for the proposed Bayesian detector. Experimental results demonstrating performance are found in Section IV, followed by conclusions in Section V. Prior conference publications providing partial results are [6] and [7].

\section{Probability Models for the Bayesian-Kalman Detector}

This section develops statistical models for the prior probability of the presence of an aircraft radar echo given the Kalman tracker history. These models will be exploited in Section II to form a new algorithm which improves the probability of detecting weak echo pulses for a given fixed rate of false alarms. The approach is founded on fundamental statistical decision theory principles and provides a unified framework for incorporating information from a Kalman tracker state space estimate into a constant false alarm rate detector. A new detection statistic, "total false alarm probability" $\left(P_{T F A}\right)$ will be introduced and used as an optimization criterion to derive the algorithm. The method is general and can be applied to any sequential detection problem possessing a dynamical state space model suitable for a Kalman tracker. The discussion here however will focus on the application of locating radar aircraft echoes in radio astronomy data.

Within a single range-azimuth bin $\left(R_{p}, \theta_{p}\right)$ consider the problem of deciding whether to accept the hypothesis that random event $H_{p}$ : "an aircraft echo is present in bin $p$ " or its complement $\bar{H}_{p}$ : "no echo is seen in bin $p$," has occurred. The associated event probabilities are $P\left(H_{p}\right)$ and $P\left(\bar{H}_{p}\right)=1-P\left(H_{p}\right)$. The classical radar detector makes this decision using a Neyman-Pearson (N-P) likelihood ratio test by comparing $b_{p}$ with a fixed threshold ${ }^{1}$ to achieve maximum probability of detection $\left(P_{D}\right)$ while enforcing a specified constraint probability of false alarm $\left(P_{F A}\right)$ [8]. This is a reasonable approach in the usual case when it is difficult to assign meaningful costs to each decision or when prior probabilities for $H_{p}$ are unavailable.

However the Kalman track state history contains information that can be interpreted, if an appropriate model can be found, as a prior probability for presence of an echo. Echoes are more likely in a region $\mathcal{S}$ surrounding a point where the tracker predicts an echo will occur, than in the rest of the range-azimuth map. The classical Bayes decision criterion seems a likely candidate to exploit this knowledge, but again we do not have the required cost measures in our radar detection problem [9]. The proposed algorithm addresses this issue and is structurally related to both the Bayes and Neyman-Pearson criteria.

\section{A. Kalman Tracker Notation}

This section reviews a few equations and notation from [4] which are necessary in developing the Bayesian detector. For simplicity the following discussion is limited to detections from a single track formed by a series of echo detections from one aircraft. However, as implemented the system manages multiple simultaneous tracks.

\footnotetext{
${ }^{1}$ When statistics for noise and echo clutter are varying, a common implementation for constant false alarm rate (CFAR) detection scales the fixed threshold by a local estimate of noise variance. This is effectively a time varying threshold detector, but does not adjust the threshold according to probability of echo presence as does the proposed algorithm.
} 
Assume the $k^{\text {th }}$ radar transmit antenna rotational pass over the radio telescope occurs nominally at time $t[k]$. This yields an aircraft echo detection, referred to as "snapshot $k$." All quantities that are updated on a once-per-snapshot basis will be represented with a $[k]$ argument. For example, the sampled position for a single aircraft detected in snapshot $k$ is expressed as $(R, \theta)[k]$ or $(x, y)[k]$.

The desired tracker outputs at snapshot $k$ are: 1$)$ a prediction point, $(\hat{x}, \hat{y})[k+1 \mid k]$, where the next detection is expected, and 2) shape parameters for region $\mathcal{S}$ centered on this point (see Figure 2 and Section II-B). $\mathcal{S}$ specifies an area likely to contain the aircraft echo during succeeding snapshot $k+1$. The "hat" notation, ^, will be used throughout to indicate an estimated or predicted quantity. An argument $[k+1 \mid k]$ indicates a predicted value for snapshot $k+1$ based on observations up to and including $k$, while $[k \mid k]$ indicates a "correction" or smoothed estimate of the true value at time $k$ based on observations through snapshot $k$. The size of $\mathcal{S}$ depends on the quality of the track and gets larger with an increase in observation noise, missed snapshot detections, or rapid acceleration of the target. $\mathcal{S}$ is also used to define the blanked region for predictive real-time blanking, to determine which track each new detection is associated with, and as the boundary for the region of increased prior probability for an arriving echo pulse used in the Bayesian detection scheme.

For an existing track, each new observation snapshot initiates an iteration of the Kalman filter which produces an updated state vector prediction, $\hat{\boldsymbol{x}}[k+1 \mid k]$, and prediction error covariance matrix $\boldsymbol{P}[k+1 \mid k]$ :

$$
\begin{aligned}
& \hat{\boldsymbol{x}}[k+1 \mid k]=\boldsymbol{F} \hat{\boldsymbol{x}}[k \mid k] \\
& \boldsymbol{P}[k+1 \mid k]=\boldsymbol{F} \boldsymbol{P}[k \mid k] \boldsymbol{F}^{T}+\boldsymbol{G} \boldsymbol{Q} \boldsymbol{G}^{T} \text {, where } \\
& \boldsymbol{x}[k]=\left[\begin{array}{llll}
x[k] & y[k] & \dot{x}[k] & \dot{y}[k]
\end{array}\right]^{T}
\end{aligned}
$$

and where $x$ and $y$ are a single aircraft's coordinates, $\dot{x}$ and $\dot{y}$ are corresponding velocities, $\boldsymbol{F}$ is the state transition matrix, $\boldsymbol{G}$ is the process transfer matrix, $\boldsymbol{Q}$ is the process acceleration matrix, and ${ }^{T}$ denotes matrix transpose. Further details are found in [4].

The prediction point $(\hat{x}, \hat{y})[k+1 \mid k]$ is given by the first two elements of $\hat{\boldsymbol{x}}[k+1 \mid k]$ in (1). Its accuracy is gauged using $\boldsymbol{P}[k+1 \mid k]$ from (2). With this prior information, it is reasonable to assume that for the snapshot at time $t[k+1]$, echo detections have higher probability near the prediction point.

\section{B. Echo Arrival Probability Model}

We propose using uncertainty region $\mathcal{S}$ as the basis for a spatial prior probability density function (pdf) model for the presence of an aircraft echo in snapshot $k+1$. A few terms must first be defined before this model can be introduced.

Assume that for each existing track exactly one echo will occur (with probability one) during snapshot $k+1$ somewhere in an annular slice region

$$
\Omega=\left\{\begin{array}{l|l}
(x, y) & \begin{array}{c}
x=R \cos \theta \text { and } y=R \sin \theta \text { where } \\
R_{\min } \leq R \leq R_{\max }, \theta_{\min } \leq \theta \leq \theta_{\max }
\end{array}
\end{array}\right\}
$$


containing all range-azimuth bins of interest. The $\{(x, y) \mid$ expression $\}$ notation defines the set of all $(x, y)$ pairs satisfying the expression. The single echo assumption implies that as far as the probability model is concerned, an established track will always produce an aircraft echo during each new antenna pass as long as the track remains in $\Omega$. Cases of aircraft dropping out of the airspace due to landing or other events are neglected. These contingencies are handled by track management logic which will drop a track after a series of snapshots which produce no detections [4].

Prediction region $\mathcal{S}$ is defined by the quadratic matrix form for an ellipse

$$
\mathcal{S}=\left\{(x, y) \mid[x-\hat{x}, y-\hat{y}] \boldsymbol{P}^{-1}[x-\hat{x}, y-\hat{y}]^{T} \leq \beta^{2} \text { and }(x, y) \in \Omega\right\}
$$

where $\in$ denotes "element of," and $(\hat{x}, \hat{y})=(\hat{x}, \hat{y})[k+1 \mid k]$ and $\boldsymbol{P}=\boldsymbol{P}[k+1 \mid k]$ are used with the snapshot dependence dropped to simplify notation. $\mathcal{S}$ is centered on $(\hat{x}, \hat{y})[k+1 \mid k]$ and has size, elongation, and orientation determined by $\boldsymbol{P}[k+1 \mid k]$. User defined parameter $\beta$ controls the scale (size) of $\mathcal{S}$ relative to the error variances in $P$.

Larger prediction error variance in $\boldsymbol{P}[k+1 \mid k]$ leads to a larger $\mathcal{S}$, representing increased uncertainty as to where the next radar echo will be detected. Figure 2 illustrates this behavior. The plot shows track evolution for real GBT data over five snapshots for a dense scene with multiple overlapping aircraft tracks. The ellipses represent $\mathcal{S}$ for each established track. Note the variety of sizes corresponding to variations in track quality.

Due to the assumption of a single echo per track - per rotational pass of the transmit antenna, we can interchangeably refer to the presence of an aircraft or its associated echo. Based on the antenna directional beamwidth (including sidelobes) and the transmit pulse length, shape and repetition rate, this echo spans many range-azimuth map bins covering many transmit pulses. Note that in this definition an echo is a collection of individual pulse returns, not just one pulse reflection. The echo thus has a footprint $F$, or region of support, due to the effective point spread function in the range-azimuth map as can be seen in Figure 1. This footprint has fixed size in $(R, \theta)$ dependent on transmit beampattern and pulse shape. The echo may or may not be detected in any of these bins, depending on echo amplitude, local noise sample statistics, and the detection algorithm. Figure 4 illustrates the geometry of $\mathcal{S}, F$, and a range-azimuth bin in $(R, \theta)$ and $(x, y)$ coordinate systems.

Let $f_{e}(x, y \mid \hat{x}, \hat{y}, \boldsymbol{P})$ be the conditional density over $(x, y)$ for the echo footprint centroid point (i.e. the actual aircraft position) given parameters defining $\mathcal{S}$. The probability the centroid is contained in some arbitrary 2-D patch $A$ is then given by $\iint_{A} f_{e}(x, y \mid \hat{x}, \hat{y}, \boldsymbol{P}) d x d y$. We propose the following pdf mixture model involving a truncated 2-D Gaussian term

$$
\begin{aligned}
& f_{e}(x, y \mid \hat{x}, \hat{y}, \boldsymbol{P})= \\
& \begin{cases}\frac{1-P_{\mathcal{S}}}{C_{\Omega} \sqrt{x^{2}+y^{2}}} & (x, y) \in \mathcal{S} \\
+\frac{1}{2 \pi \sigma^{2}|\boldsymbol{P}|} \exp \left(-\frac{1}{2 \sigma^{2}}[x-\hat{x}, y-\hat{y}] \boldsymbol{P}^{-1}[x-\hat{x}, y-\hat{y}]^{T}\right) & \\
\frac{1-P_{\mathcal{S}}}{C_{\Omega} \sqrt{x^{2}+y^{2}}} & (x, y) \in \overline{\mathcal{S}}\end{cases}
\end{aligned}
$$


The notation $\overline{\mathcal{S}}$ indicates the complement of region $\mathcal{S}$ such that $\Omega=\mathcal{S} \cup \overline{\mathcal{S}}$ where $\cup$ is set union. The notation $f(\cdot)$ will be used throughout the paper to denote any pdf, and subscript $e$ here identifies this as an echo centroid distribution over space. Figure 3 presents an example realization for this pdf.

User defined parameter $\sigma$ controls how concentrated $f_{e}(x, y \mid \hat{x}, \hat{y}, \boldsymbol{P})$ is around its central peak. The remaining terms in (4) are defined as

$$
\begin{aligned}
P_{\mathcal{S}} & =\iint_{\mathcal{S}} \frac{1}{2 \pi \sigma^{2}|\boldsymbol{P}|} \exp \left(-\frac{1}{2 \sigma^{2}}[x-\hat{x}, y-\hat{y}] \boldsymbol{P}^{-1}[x-\hat{x}, y-\hat{y}]^{T}\right) d x d y \\
& =1-e^{-\frac{\beta^{2}}{2 \sigma^{2}}} \\
C_{\Omega} & =\iint_{\Omega} \frac{1}{\sqrt{x^{2}+y^{2}}} d x d y,=\iint_{\Omega} d R d \theta \\
& =\left(R_{\max }-R_{\min }\right)\left(\theta_{\max }-\theta_{\min }\right)
\end{aligned}
$$

where $|\boldsymbol{P}|$ is the matrix determinant of $\boldsymbol{P}$. Equation (5) was evaluated by substitution of (3) for $\mathcal{S}$, using $x=\alpha \rho \cos \theta$ and $y=\rho \sin \theta$, setting $(\hat{x}, \hat{y})=(0,0)$, and solving in polar coordinates to obtain (6). The probability the echo centroid is anywhere within $\mathcal{S}$ is given by $P\left(H_{\mathcal{S}}\right)=\iint_{\mathcal{S}} f_{e}(x, y \mid \cdot) d x d y=\iint_{\mathcal{S}} \frac{1-P_{\mathcal{S}}}{C_{\Omega} \sqrt{x^{2}+y^{2}}} d x d y+P_{\mathcal{S}} \approx P_{\mathcal{S}}$. The approximation is due to the Gaussian term in (4) dominating the expression. Outside of $\mathcal{S}$ we assume each rangeazimuth bin is equally likely to contain the echo centroid. This assumption is made to force a constant threshold, and thus conventional constant $P_{F A}$ performance outside of $\mathcal{S}$ in the Bayesian detection framework introduced in the following section.

The area of a bin is proportional to $R$, which necessitates that over $\overline{\mathcal{S}}, f_{e}(x, y \mid \hat{x}, \hat{y}, \boldsymbol{P})$ must be proportional to $1 / \sqrt{x^{2}+y^{2}}=1 / R$. Dividing by $C_{\Omega}$ in (4) normalizes the integral to this bin area growth with $R$. Problems with division by zero are avoided since in every practical case $R_{\min } \gg 1$. Note also that this definition insures that $\iint_{\Omega} f_{e}(x, y \mid \hat{x}, \hat{y}, \boldsymbol{P}) d x d y=1$ as required for a pdf.

This model is a reasonable choice because it possesses the following necessary characteristics:

- $f_{e}(x, y \mid \hat{x}, \hat{y}, \boldsymbol{P})$ decreases monotonically with distance from the prediction point.

- Over $\mathcal{S}$ it is a smooth function with no discontinuities.

- The shape of $f_{e}(x, y \mid \hat{x}, \hat{y}, \boldsymbol{P})$ depends on prediction error covariance, $\boldsymbol{P}[k+1 \mid k]$, such that higher prediction error leads to lower probability density.

- There is a low, uniform probability across all range-azimuth bins outside the prediction region for presence of the echo centroid.

\section{Calculating Prior Probability $P\left(\bar{H}_{p}\right)$}

Given the echo centroid pdf model, $f_{e}(x, y \mid \hat{x}, \hat{y}, \boldsymbol{P})$, it is possible to solve for the probability $P\left(H_{p}\right)$ that the echo footprint intersects range-azimuth bin $p$.

Let $A_{p}$ be the 2-D patch which covers bin $p$ and which has midpoint $\left(x_{p}, y_{p}\right)=\left(R_{p} \cos \theta_{p}, R_{p} \sin \theta_{p}\right)$. Uniform sampling in $R_{p}$ and $\theta_{p}$ yields non uniform spacing in $(x, y)$, so the size and orientation of $A_{p}$ depend on $R_{p}$ and 
$\theta_{p}$ as shown in Figure 4, and must be considered when computing $P\left(H_{p}\right)$. Define $F_{q}$ as the footprint region for an echo with centroid at another arbitrary bin $q$. All bins $q$ where $F_{q}$ intersects $A_{p}$ contribute to the probability $P\left(H_{p}\right)$ of an echo in bin $p$. The probability that the echo centroid is in bin $q$ is given by $\iint_{A_{q}} f_{e}(x, y \mid \hat{x}, \hat{y}, \boldsymbol{P}) d x d y$. Thus the probability that any portion of the echo footprint is contained in bin $p$ is given by

$$
P\left(H_{p}\right)=\sum_{\left\{q \mid A_{p} \cap F_{q} \neq \phi\right\}} \iint_{A_{q}} f_{e}(x, y \mid \hat{x}, \hat{y}, \boldsymbol{P}) d x d y
$$

where $\phi$ is the empty set and $\cap$ denotes intersection. Point $\left(x_{p}, y_{p}\right)$ is at the approximate centroid of a region $\breve{F}_{p}$ defined as the area covered by the union of all $F_{q}$ that satisfy $A_{p} \cap F_{q} \neq \phi$, i.e. all $F_{q}$ that overlap $A_{p}$.

When $\breve{F}_{p}$ is small ${ }^{2}$ compared to $\mathcal{S}$ the local surface of $f_{e}(x, y \mid \hat{x}, \hat{y}, \boldsymbol{P})$ around $\left(x_{p}, y_{p}\right)$ is well approximated by a plane. Since all bin patches $A_{q}$ in this region are approximately the same size as $A_{p}$, and the average of a function of constant slope over a symmetric planar region is found at its centroid, (7) can be approximated as

$$
\begin{aligned}
P\left(H_{p}\right) & \approx \sum_{\left\{q \mid A_{p} \cap F_{q} \neq \phi\right\}} \iint_{A_{p}} f_{e}(x, y \mid \hat{x}, \hat{y}, \boldsymbol{P}) d x d y \\
& \approx \sum_{\left\{q \mid A_{p} \cap F_{q} \neq \phi\right\}} \alpha_{p} f_{e}\left(x_{p}, y_{p} \mid \hat{x}, \hat{y}, \boldsymbol{P}\right) \\
& \approx K \alpha_{p} f_{e}\left(x_{p}, y_{p} \mid \hat{x}, \hat{y}, \boldsymbol{P}\right)
\end{aligned}
$$

where $\alpha_{p}=\iint_{A_{p}} d x d y$ is the area of patch $A_{p}$ and $K$ is the number of bins, indexed by $q$, satisfying $\left\{q \mid A_{p} \cap F_{q} \neq\right.$ $\phi\}$. The approximations are due to integrating over $A_{p}$ rather than $A_{q}$ and assuming $f_{e}(x, y \mid \hat{x}, \hat{y}, \boldsymbol{P})$ is constant over small patch $A_{p}$ in (8).

Thus substituting (4) into (10) yields

$$
\begin{aligned}
& P\left(H_{p}\right) \approx\left\{\begin{array}{lr}
\frac{K \alpha_{p}\left(1-P_{\mathcal{S}}\right)}{C_{\Omega} \sqrt{x_{p}^{2}+y_{p}^{2}}} & \left(x_{p}, y_{p}\right) \in \mathcal{S} \\
+\frac{K \alpha_{p}}{2 \pi \sigma^{2}|\boldsymbol{P}|} \exp \left(-\frac{1}{2 \sigma^{2}}\left[x_{p}-\hat{x}, y_{p}-\hat{y}\right] \boldsymbol{P}^{-1}\left[x_{p}-\hat{x}, y_{p}-\hat{y}\right]^{T}\right) & \\
& \\
\frac{K \alpha_{p}\left(1-P_{\mathcal{S}}\right)}{C_{\Omega} \sqrt{x_{p}^{2}+y_{p}^{2}}} & \left(x_{p}, y_{p}\right) \in \overline{\mathcal{S}}
\end{array}\right. \\
& P\left(\bar{H}_{p}\right)=1-P\left(H_{p}\right) .
\end{aligned}
$$

The specific pulse repetition and antenna sweep rates of the ARSR-3 radar as observed at the GBT, coupled with our baseband sample rate for $b[n]$, lead to range-azimuth bin widths of $\Delta_{R} \approx 13.87 \mathrm{~m}$ and $\Delta_{\theta} \approx 0.0015$ radians. This yields $\alpha_{p} \approx \Delta_{\theta} \Delta_{R} R_{p}=0.0208 R_{p}$.

\footnotetext{
${ }^{2}$ We have found that $\breve{F}_{p}$ is usually smaller than $\mathcal{S}$ in practice, due to the short range extent of echoes, and particularly when only the transmit beampattern mainlobe is considered to be part of the echo footprint $F_{q}$. Some very bright echoes have sidelobe extent in azimuth that could extend beyond $\mathcal{S}$ (see Figure 1), but excluding these from $F_{q}$ is reasonable since we do not wish to emphasize detection of sidelobes in any case.
} 


\section{Bayesian Detector Algorithm Development}

This section presents details of the proposed improved detection algorithm which is based on a new optimal detection criterion, "constant total false alarm probability."

\section{A. Detection Statistic Model}

The goal of radar detection is to observe digital receiver output time series $b[n]$ (equivalently $b_{p}$ ) and make a decision at each time sample (equivalently range-azimuth bin $p$ ) whether an echo is present. For a single bin $p, b_{p}$ is a random variable with conditional amplitude pdf's $f_{b}\left(b_{p} \mid \bar{H}_{p}\right)$ and $f_{b}\left(b_{p} \mid H_{p}\right)$, given the absence or presence of an echo respectively. Note that while $f_{e}(x, y \mid \cdot)$ is a distribution over space, $f_{b}\left(b_{p} \mid \cdot\right)$ describes an amplitude, or voltage distribution.

Digital receiver output $b_{p}$ is formed as the sum of the squares of the real (in phase) and imaginary (quadrature) baseband matched filter channels, each of which contains independent additive Gaussian noise [10]. As such, $b_{p}$ consists of the sum of two squared Gaussian random variables, so $b_{p} / \sigma_{\eta}$ (where $\sigma_{\eta}^{2}$ is the noise variance for both the real or imaginary channels) has a central chi-squared $\left(\chi_{2}^{2}\right)$ distribution with two degrees of freedom under $\bar{H}$, and noncentral $\chi_{2}^{2}$ under $H$ as illustrated in Figure 5 [8][11]. In many communications texts $v_{p}=\sqrt{b_{p}}$ is used as the detection statistic, and it is shown to have a Rice distribution,

$$
f_{v}\left(v_{p} \mid H_{p}\right)=\frac{v_{p}}{\sigma_{\eta}^{2}} I_{0}\left(\frac{v_{p}|s|}{\sigma_{\eta}^{2}}\right) e^{-\frac{v_{p}^{2}+s^{2}}{2 \sigma_{\eta}^{2}}}
$$

where $I_{0}(\cdot)$ is the zero-th order modified Bessel function of the first kind and noncentrality parameter $s$ is proportional to echo signal power [10]. The following discussion uses $b_{p}$ as the detection statistic, but a parallel development could also be presented using $v_{p}$ by simply taking the square root of each side of every threshold test inequality.

\section{B. Related Detector Structures}

This section discusses some well known detector architectures as a brief tutorial background and to motivate design choices for the new method. The intent is to show that the Bayesian constant $P_{T F A}$ detector introduced in equations (19) - (21) below can be interpreted as a natural extension of familiar concepts.

The famous Neyman-Pearson (N-P) detector uses the following likelihood ratio test:

$$
\frac{f_{b}\left(b_{p} \mid H_{p}\right)}{f_{b}\left(b_{p} \mid \bar{H}_{p}\right)} \stackrel{\stackrel{H}{>}}{\underset{H}{<}} \tau_{\mathrm{NP}}^{\prime}
$$

where the stacked inequality denotes "decide $H_{p}$ if the left hand side is greater than $\tau_{\mathrm{NP}}^{\prime}$, otherwise choose $\bar{H}_{p}$." Since the likelihood ratio is monotonic in $b$ for these particular pdf's, (13) is equivalent to a simple direct thresholding of the receiver output:

$$
b_{p} \stackrel{\stackrel{H}{>}}{>} \tau_{\mathrm{NP}}
$$


where $\tau_{\mathrm{NP}}$ is a function of the constant threshold $\tau_{\mathrm{NP}}^{\prime}$. The associated probability of false alarm (detecting an echo when none is present) is defined as

$$
P_{F A}=\int_{\tau_{\mathrm{NP}}}^{\infty} f_{b}\left(b_{p} \mid \bar{H}_{p}\right) d b .
$$

The usual approach for establishing the threshold level is to fix $P_{F A}$ to some suitably small constant and then solve (15) for $\tau_{\mathrm{NP}}$. Note that $P_{F A}$ is defined conditionally under $\bar{H}$ and that the integrand comes from the denominator of the left hand side of (13).

The simple NP test does not provide a mechanism for exploiting prior probability information obtained from the tracker. One possible alternative is the Bayes decision criterion [9] [12]

$$
\frac{P\left(H_{p}\right) f_{b}\left(b_{p} \mid H_{p}\right)}{P\left(\bar{H}_{p}\right) f_{b}\left(b_{p} \mid \bar{H}_{p}\right)} \underset{\bar{H}}{>} \frac{C_{H, \bar{H}}-C_{\bar{H}, \bar{H}}}{C_{\bar{H}, H}-C_{H, H}}=\tau(C)
$$

where constants $C_{I, J}$ for $I, J \in\{\bar{H}, H\}$ are costs (also called Bayes loss) associated with deciding $I$ when $J$ is true, and $C=\left\{C_{\bar{H}, \bar{H}}, C_{\bar{H}, H}, C_{1, \bar{H}}, C_{H, H}\right\}$. Unfortunately, (16) has some disadvantages. We do not have a satisfactory systematic approach to specify the costs. Also it is not clear how to frame this in the desired direct thresholding form of (14).

To illustrate the difficulty, note that the left hand side of (16) is still monotonic in $b_{p}$ and that $\tau(C)$ is a constant (though dependent on $C$ ). This suggests that a corresponding threshold test comparable to (13) would be

$$
\frac{f_{b}\left(b_{p} \mid H_{p}\right)}{f_{b}\left(b_{p} \mid \bar{H}_{p}\right)} \underset{\bar{H}}{\stackrel{H}{>}} \frac{P\left(\bar{H}_{p}\right)}{P\left(H_{p}\right)} \tau(C)=\tau(p, C) .
$$

Now threshold $\tau(p, C)$ depends on position $p$ since $P\left(\bar{H}_{p}\right)$ and $P\left(H_{p}\right)$ are spatially varying. In this Bayesian framework the direct threshold form corresponding to (14) is found by defining the likelihood ratio function $L(b)=$ $f_{b}\left(b \mid H_{p}\right) / f_{b}\left(b \mid \bar{H}_{p}\right)$ and operating on the left and right hands sides of (17) with the functional inverse $L^{-1}(\cdot)$ to obtain

$$
b_{p} \underset{\bar{H}}{\stackrel{H}{>}} L^{-1}(\tau(p, C))=\tau^{\prime}(p, C) .
$$

The problem with this formulation is that although it is in fact the desired direct test on receiver output sample $b_{p}$, we do not know how to evaluate threshold $\tau^{\prime}(p, C)$. In a typical scenario all the $C_{I, J}$ are unknown. Also $L^{-1}(\cdot)$ depends on $f_{b}\left(\tau(p, C) \mid H_{p}\right)$ which unlike $f_{b}\left(\tau(p, C) \mid \bar{H}_{p}\right)$ is hard to model because aircraft echoes have unknown random intensities. One cannot readily find the appropriate threshold value because we cannot use the constant $P_{F A}$ constraint of (15) to avoid these complexities and still satisfy the desired Bayesian relationships of (16).

\section{Constant $P_{T F A}$ Detection}

We propose an alternative Bayesian framework for improved detection performance to address the problems mentioned above, and which is based on a physically meaningful criterion related to specifying a fixed $P_{F A}$. We 
define the unconditional "total probability of false alarm" $\left(P_{T F A}\right)$ as the joint probability that a spatially varying threshold, $\tau_{p}$, was crossed and no echo was present,

$$
\begin{aligned}
P_{T F A} & =P\left(\left(b_{p}>\tau_{p}\right) \cap \bar{H}_{p}\right) \\
& =\int_{\tau_{p}}^{\infty} P\left(\bar{H}_{p}\right) f_{b}\left(b \mid \bar{H}_{p}\right) d b \\
& =P\left(\bar{H}_{p}\right) P_{F A} .
\end{aligned}
$$

This is the total probability (i.e. not conditioned on $H$ or $\bar{H}$ ) that a false alarm will occur at range-azimuth bin $p$ given some $\tau_{p}$.

Equation (19) provides a means to solve for an appropriate threshold for a direct test in the form of (14) or(18), but which incorporates the prior information $P\left(\bar{H}_{p}\right)$ drawn from the Kalman track history through equations (11) and (12). Note that $\tau_{p} \neq \tau^{\prime}(p, C) \neq \tau_{\mathrm{NP}}$ so the detector is neither N-P, nor classical Bayes decision criterion, but depends on the Bayes relationship used in (19).

Spatially varying $\tau_{p}$ is found by specifying a fixed value for the acceptable $P_{T F A}$ and solving (19) for $\tau_{p}$ in closed form:

$$
\tau_{p}=-\sigma_{\eta}^{2} \log \left(\frac{P_{T F A}}{P\left(\bar{H}_{p}\right)}\right)
$$

where $P_{T F A}$ is specified by the user to be some small constant, $P\left(\bar{H}_{p}\right)$ is given by (11) and (12), and $2 \sigma_{\eta}^{2}$ is the complex noise variance for $b[n]$.

This is not the same test as in (16). One obvious distinction is that costs $C_{i, j}$ are not included since the threshold is set by solving a $P_{T F A}$ equation. Some insight is provided by noting that (19) uses the left hand side denominator of (16) as an integrand, just as (15) integrates the denominator of (13). Also, we propose that constant $P_{T F A}$ detection is a rational solution given that the expected total number of false alarms will be known over the range azimuth map. This is set as a design parameter. On the other hand, a fixed $P_{F A}$ detector will have varying numbers of false alarms as $P(\bar{H})$ varies. The computational load for a radar detection system is proportional to the number of threshold crossings, so being able to fix the average number of false alarms can be a useful constraint on system capacity.

Summarizing, the constant $P_{T F A}$ detector (CTFA) is computed with the following steps:

1) Set $P_{T F A}=$ a small constant.

2) Evaluate (20) to find the spatially varying $\tau_{p}$ at each range-azimuth bin, $b\left(x_{p}, y_{p}\right)$.

3) Threshold each bin:

$$
b_{p} \stackrel{\stackrel{H}{>}}{\underset{H}{\leftarrow}} \tau_{p} .
$$

The effect of this approach is that the threshold is lowered in the prediction regions $\mathcal{S}$ where based on prior track history it is known that echoes are more likely. This leads to more reliable detection of weak aircraft echoes. By proper selection of parameters $P_{T F A}, \beta$, and $\sigma$, the probability of detection $P_{D}=\int_{\tau_{p}}^{\infty} f_{b}\left(b_{p} \mid H_{p}\right)$ is increased while 
false alarms are kept low, as shown in Figure 5. Figure 6 illustrates the variable threshold $\tau_{p}$ plotted as a function of $x$ and $y$ for two prediction regions from two tracks. Note that the lower quality track has a wider prediction region (right), leading to a more shallow threshold depression. This corresponds to greater uncertainty about the next echo location, so less emphasis on detection is generated here. Note however that in both cases the threshold reduction is relatively small (less than $0.5 \%$ in this example) as a natural consequence of the way the constant $P_{T F A}$ detector is defined. However, since it is applied over many bins, even such a small change in threshold can have a significant effect on detection statistics, improving the probability of finding a threshold crossing in at least one bin of the echo footprint. This is demonstrated in results presented in the following section. With this small threshold depression $P_{D}$ is increased while avoiding the potential problem of forcing a detection in $\mathcal{S}$ whether an echo is present or not.

As noted earlier there are often multiple concurrent aircraft tracks in $\Omega$, each with a prediction region and associated computed threshold levels $\tau_{p}$. This potential conflict is handled as follows. Let $\tau_{p, j}$ be the threshold computed for the $p^{\text {th }}$ range-azimuth bin using $\mathcal{S}_{j}$ from the $j^{\text {th }}$ track. A single threshold test is made for each bin as in (21) where

$$
\tau_{p}=\min _{j} \tau_{p, j} .
$$

This method was used in Figure 6. The set of threshold crossings is then processed with the CLEAN algorithm [13] to collapse the sidelobe structure to a single point, and each cleaned detection is assigned to an existing or new track following arbitration rules described in [4].

\section{EXPERIMENTAL RESUlTS}

\section{A. Performance Evaluation with Simulated Data}

It is very difficult to quantitatively characterize detection performance using real-world data because relevant signal properties such as signal to noise ratio (SNR) and absence or presence of an echo are unknown. We have developed a realistic echo synthesis code to generate data used in Monte Carlo simulation trials to estimate detection properties such as probability of detection $\left(P_{D}\right)$ and probability of false alarm $\left(P_{F A}\right)$ as functions of SNR and aircraft motion dynamics. The new Bayesian detector is shown to improve $P_{D}$ for a fixed $P_{F A}$ as compared with the conventional approach, thus validating the strategy of detector design based on constant $P_{T F A}$.

The simulator generates a time sequence $b[n]$ of receiver outputs with the following properties:

- Receiver sensitivity, IF bandwidth, data sample rate, and antenna sidelobe gain response all approximately match those used to collect radar data at the GBT.

- Transmitter pulse length, pulse shape, pulse repetition rate, antenna sweep rate, and transmit beamwidth approximate those of the ARSR-3 radar system as discussed in [14], [15], [5], [4].

- Additive white Gaussian noise (AWGN) is used with variance $\sigma_{\eta}{ }^{2}$ matching sample variance estimated from real radar-echo-free GBT data.

- In order to eliminate problems in associating new detections with their corresponding tracks, there is only one simulated echo track in each randomly generated trial observation. 
- Realistic aircraft motion is simulated using the Kalman state space model with acceleration $\boldsymbol{a}(k)$ generated as a lowpass filtered 2-D Gaussian i.i.d. random noise process. The lowpass corner frequency is adjusted to produce smoothly connected random maneuvers with turn radii consistent with commercial aircraft.

- The pulse echo amplitude follows a Swirling IV distribution model suitable for a square law receiver [16]. Received pulses have constant mean amplitude throughout an entire scan, but are uncorrelated from pulse to pulse. The echo amplitude pdf is given by

$$
f_{\Sigma}(\sigma)=\frac{4 \sigma}{\sigma_{a v}^{2}} \exp \left(-\frac{2 \sigma}{\sigma_{a v}}\right)
$$

where subscript $\Sigma$ denotes the Swirling pdf, $\sigma$ is the random radar cross section (RCS) value, and $\sigma_{a v}$ is the average RCS over all target fluctuations.

A Monte-Carlo simulation was used with repeated random trials to evaluate $P_{D}$ vs. $P_{F A}$ in order to estimate the receiver operating characteristic (ROC). When comparing the new Bayesian algorithm with a conventional detector one must carefully consider the differences in how false alarms arise. As defined in (15), $P_{F A}$ only depends on the noise power and a threshold $\tau_{\mathrm{NP}}$ with fixed value over the entire range-azimuth map. However, the new algorithm improves $P_{D}$ at the cost of a slight local false alarm rate increase inside $\mathcal{S}$. To be fair, a comparison of $P_{D}$ between algorithms must be made when each has the same effective total $P_{F A}$ value. For Figure 7 the "Probability of False Alarm" values along the horizontal axis represent actual observed error counts across a series of range-azimuth map random realizations, divided by the number of trials and the number of bins (pixels) in a map. In order to calculate sample statistics in the realistic $P_{F A}$ regime between $10^{-8}$ and $10^{-6}$ it was necessary to run many thousands of random trials to observe enough of the rare false alarms to keep error variance low. A family of ROC curves is presented to demonstrate the superior performance of the new algorithm. Detection probabilities are uniformly higher for the Bayesian detector when both algorithms are producing the same number of false alarms.

\section{B. Performance with Real Data}

Two sets of real data were recorded at the GBT and used to test performance of the echo detection algorithm, tracking, and blanking. Set one consists of five 12 second long data windows recorded at one minute intervals on April 5, 2002. Set two contains a continuous block of 10 minutes of data (50 radar antenna rotations) recorded in January, 2003. The Kalman tracker and both the conventional N-P and new Bayesian detectors were run on all data in both sets. A more extensive presentation of these results is found in [5].

In data set one there are five snapshots containing eight distinct aircraft tracks. The Bayesian detector found three echoes that were missed by the conventional method. A missed detection was declared when no conventional threshold crossing occurred in a track's prediction region at snapshot $t[k+1]$, but was reacquired in snapshot $t[k+2]$, i.e. the tracker had to make a two-step prediction update to keep the track alive. The reacquisition at $t[k+2]$ implies that the aircraft echo was present in $\mathcal{S}$ at $t[k+1]$, but was just too weak to detect. In total there were 33 track associated detections with the conventional algorithm, and 36 with the new detector. No "false alarm" detections (i.e. new threshold crossings not associated with a track) arose with the new method. 
In data set two there were four missed detections recovered by the Bayesian algorithm over 15 snapshots with six active tracks as shown in Figure 8. One more possible false alarm was made by the Bayesian detector than with the N-P approach. For this experiment both $P_{F A}$ and $P_{T F A}$ were set to $3.87 \times 10^{-8}$ when calculating threshold settings.

On the other hand, Figure 9 illustrates what occurs when the N-P detection threshold is lowered (by increasing the design $P_{F A}$ ) in an attempt to recover the lost echoes which were found by the Bayesian detector. When $P_{F A}$ is raised from $3.87 \times 10^{-8}$ to $2.32 \times 10^{-7}$ two of the four missing detections were found, but four false alarms were added. In order to detect the remaining two echoes $P_{F A}$ had to be raised so high that hundreds of false alarms appeared (not shown in this figure). Clearly the Bayesian approach provides better false alarm management while improving weak echo detection.

To illustrate the potential scientific observational impact of blanking the previously missed echoes which are now caught by the Bayesian algorithm we processed a segment of data from data set two. This window from 187.2 to 188.4 seconds in the recording produced no aircraft detections using the conventional algorithm, but the new method found three. Figure 10 shows power spectral estimates accumulated over the 1.2 second window which contained ground terrain echo clutter. The upper curve includes no radar pulse blanking and exhibits a dominant spectral peak around 5.5 MHz caused by ground clutter. The lower curve shows the spectrum after fixed time window blanking synchronized to the transmit antenna sweep. This corresponds to excising all data between a delay of 0 and 150 $\mu \mathrm{s}$ in Figure 1. At this plotting scale all RFI appears to have been eliminated. All blanking was implemented by "zero-stuffing" [3], that is, placing zeros into the time samples where radar interference is detected.

However, at the expanded scale of Figure 11, a difference is seen between spectral estimates using the new Bayesian detection as compared with conventional N-P detection. The Kalman track was established from five previous antenna sweeps, which occur at $12 \mathrm{~s}$ intervals. The three weak aircraft echoes which were detected with the new algorithm were blanked, and the resulting lower curve spectrum shows reduced bias near $5.5 \mathrm{MHz}$ corresponding to the radar pulse center frequency.

\section{CONCLUSIONS}

The forgoing analysis has demonstrated the potential for a Kalman tracker based Bayesian detector to provide high performance pulsed interference removal from radio astronomical data. Even when resulting spectral estimates are improved only subtly, it is desirable in radio astronomy to remove every interference component possible to increase confidence in the affected scientific observation. These radar systems are not going away, and as interest increases for deep space observation in the radar band allocations such a system will become increasingly necessary.

Though the mathematical development presented here appears fairly complex, computer implementation of the tracker - constant $P_{T F A}$ detector is quite straightforward. Computational burdens are small because track state and threshold computation updates need be made only once per 12 second antenna sweep. Thus, aside from the digital receiver functions, only modest computational resources are required as long as a digitized signal stream is available. The digital radar receiver must operate at RF sample rates, but such systems are in widespread use. 
Any system used to blank pulses after detecting them (e.g. using N-P detection) must use the same type digital radar receiver front-end. Therefore, by comparison the additional computational burden to add Kalman tracking and constant $P_{T F A}$ detection could be handled by the slowest of PC's.

This study suggests that the Kalman tracker Bayesian detector system is a good candidate for real-time operation and permanent implementation at the observatory. Plans are currently under consideration to pursue funding for such an obvious next step.

However, even without investing in significant real-time processing resources the proposed system can be used in a post processing mode on virtually any extended pre-recorded data set containing pulsed radar RFI. In a post processing scenario it would also be possible to adapt the proposed approach to operate both backwards and forwards in time. One can envision a forward-backward two pass echo Kalman tracking scheme and a Bayesian detector which draws on the entire data set (rather than just past detections) for prior probability inference. This should offer additional detection performance gains, particularly at the beginning of an aircraft track where start-up errors are large. As described above, two successive N-P detections are required before a track can be initiated. Using backward prediction it would be possible to find weak echoes which did not cross the N-P threshold and which occurred before the causal track was created. Future research plans include developing this bidirectional tracking detector. Block estimation methods that do not rely on sequential processing, either forward or backward, can also be investigated.

\section{REFERENCES}

[1] S. Ellingson and G. Hampson, "Rfi and asynchronous pulse blanking in the 1230-1375 mhz band at arecibo," Ohio State University, Tech. Rep. 743467-3, 2003.

[2] S. Ellingson, "Capabilities and limitations of adaptive canceling for microwave radiometry," IEEE International Geoscience and Remote Sensing Symposium 2002, IGARSS'02, vol. 3, pp. 1685-1687, June 2002.

[3] Q. Zhang, Y. Zheng, S. Wilson, J. Fisher, and R. Bradley, "Combating pulsed radar interference in radio astronomy," The Astronomical Journal, vol. 126, pp. 1588-1594, 2003.

[4] W. Dong, B. Jeffs, and J. Fisher, "Radar interference blanking in radio astronomy using a kalman tracker," Radio Science, RS5S04, doi:10.1029/2004RS003130, vol. 40, no. 5, June 2005.

[5] W. Dong, "Time blanking for gbt data with radar rfi," Master's thesis, Brigham Young University, 2004.

[6] W. Dong, B. Jeffs, and J. Fisher, "A kalman-tracker-based bayesian detector for radar interference in radio astronomy," in Proc. of the IEEE International Conf. on Acoust,, Speech, and Signal Processing, vol. IV, 2005, pp. 657-660.

[7] W. Dong and B. Jeffs, "Kalman tracking and bayesian detection for radar rfi blanking," in Proceedings of RFI2004, IUCAD DRAO Workshop in Mitigation of Radio Frequency Interference in Radio Astronomy, Web address: http://www.ece.vt.edu/ swe/RFI2004/, Penticton, British Columbia, July 2004.

[8] H. V. Trees, Detection, Estimation, and Modulation Theory, Part III, Radar-Sonar Signal Processing and Gaussian Signals in Noise. John Wiley and Sons, 2001.

[9] —_, Detection, Estimation, and Modulation Theory, Part I. John Wiley and Sons, 2001.

[10] J. Proakis and M. Salehi, Communications Systems Engineering, Second Ed. Englewood Cliffs, New Jersey: Prentice Hall, 2002.

[11] A. Papoulis, Probability, Random Variables, and Stochastic Processes, Second Ed. New-York: McGraw Hill, 1984.

[12] L. L. Scharf, Statistical Signal Processing: Detection, Estimation, and Time Series Analysis. Reading, Massachusetts: Addison-Wesley, 1991.

[13] J. Högbom, "Aperture synthesis with a nonregular distribution of interferometer baselines," Astronomy and Astrophysics Supplement, vol. 15, pp. 417-426, 1974. 
[14] J. Fisher, "Summary of rfi data samples at green bank," National Radio Astronomy Observatory, Green Bank Observatory, Tech. Rep., 2001

[15] _ _ "Analysis of radar data from february 6, 2001," National Radio Astronomy Observatory, Green Bank Observatory, Tech. Rep., 2001.

[16] B. Mahafza, Radar Systems Analysis and Design Using MATLAB. Chapman and Hall/CRC Press, 2000. 


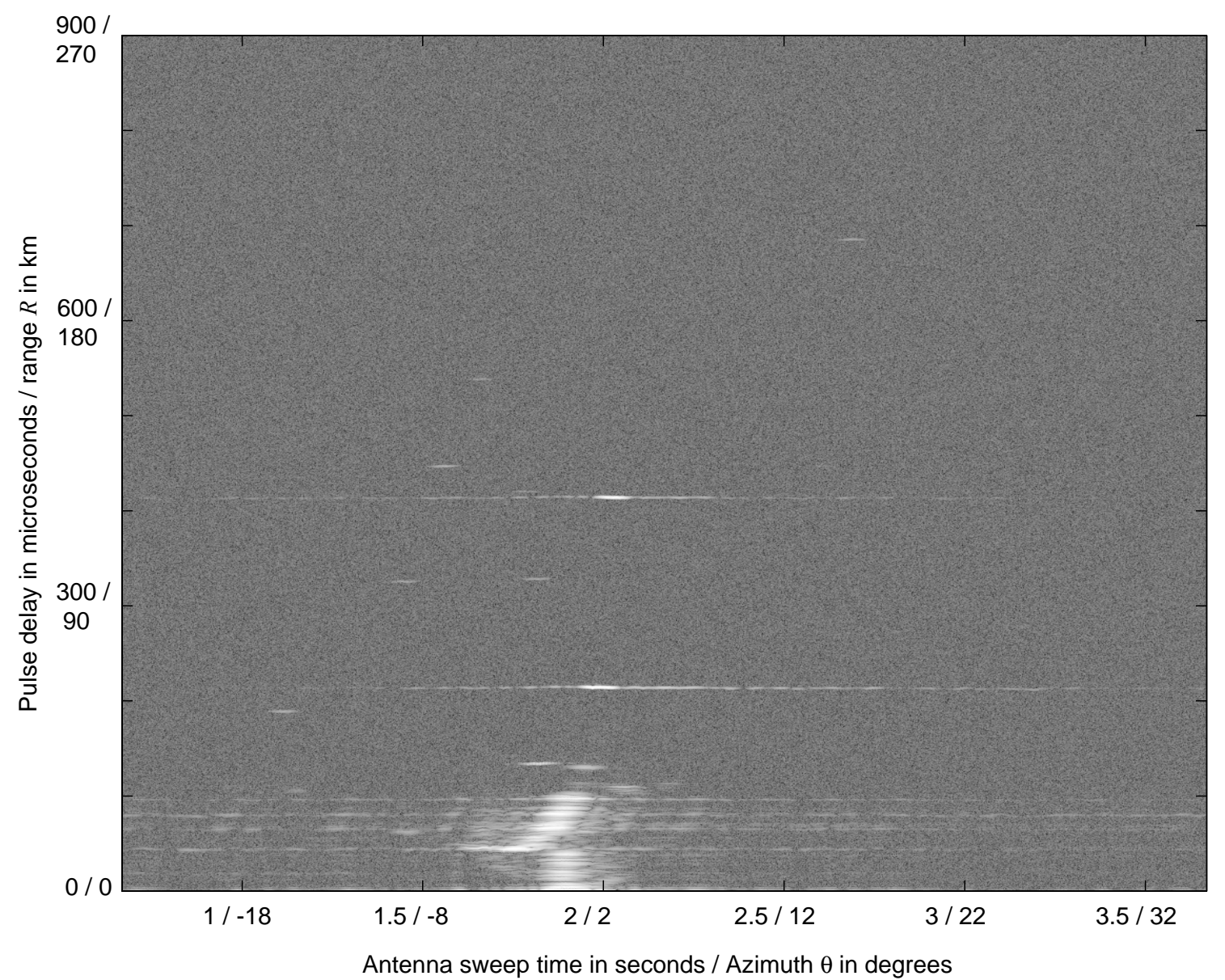

Fig. 1. A typical range-azimuth map, $b(R, \theta)$, of radar data collected at the GBT. This map covers approximately $60^{\circ}$ of azimuth $\theta$ along the $3.5 \mathrm{~s}$ span of the horizontal axis. The bright region at about 1.8 seconds and 0-100 $\mu$ s delay corresponds to the transmitter beam passing overhead at the GBT and generating strong reflections from nearby mountain peaks. Several aircraft echoes are visible. Note the horizontal extent of the bright echoes due to the sidelobe directional response pattern of the radar transmit antenna. The echo extent or region of support for a single aircraft is denoted as footprint $F$. 


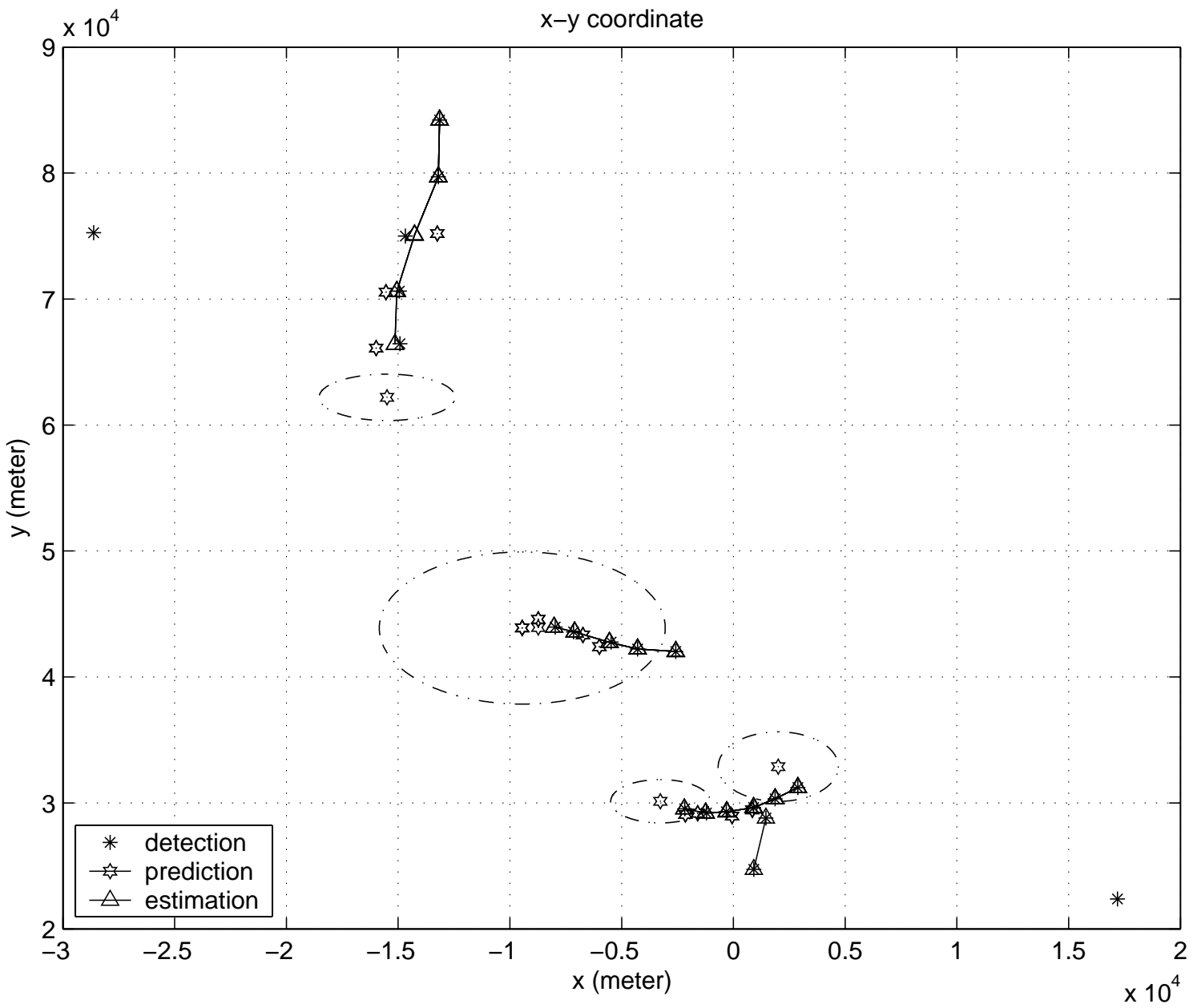

Fig. 2. An example of Kalman tracking performance for data acquired at the GBT. Four aircraft tracks have been automatically established and plotted, including a pair of crossing tracks. Data from five snapshots are shown. In order to exaggerate the motion between snapshots and reduce computational demand, only every other antenna pass was used, resulting in a snapshot interval $\approx 24 \mathrm{~s}$. The final point plotted for each track is the prediction point, $(\hat{x}, \hat{y})[k+1 \mid k]$. Prediction regions, $\mathcal{S}$, shown by the dashed ellipses vary in size according to track quality. Note that the center track has a large $\mathcal{S}$ due to a missed detection. The entire area covered by the figure is represented by $\Omega$. Predictive real-time blanking is accomplished by excising the prediction region data. For this example, off diagonal terms in $\boldsymbol{P}[k+1 \mid k]$ were assumed to be zero, which forces the $\mathcal{S}$ ellipses to align to the $x$ and $y$ axes. 


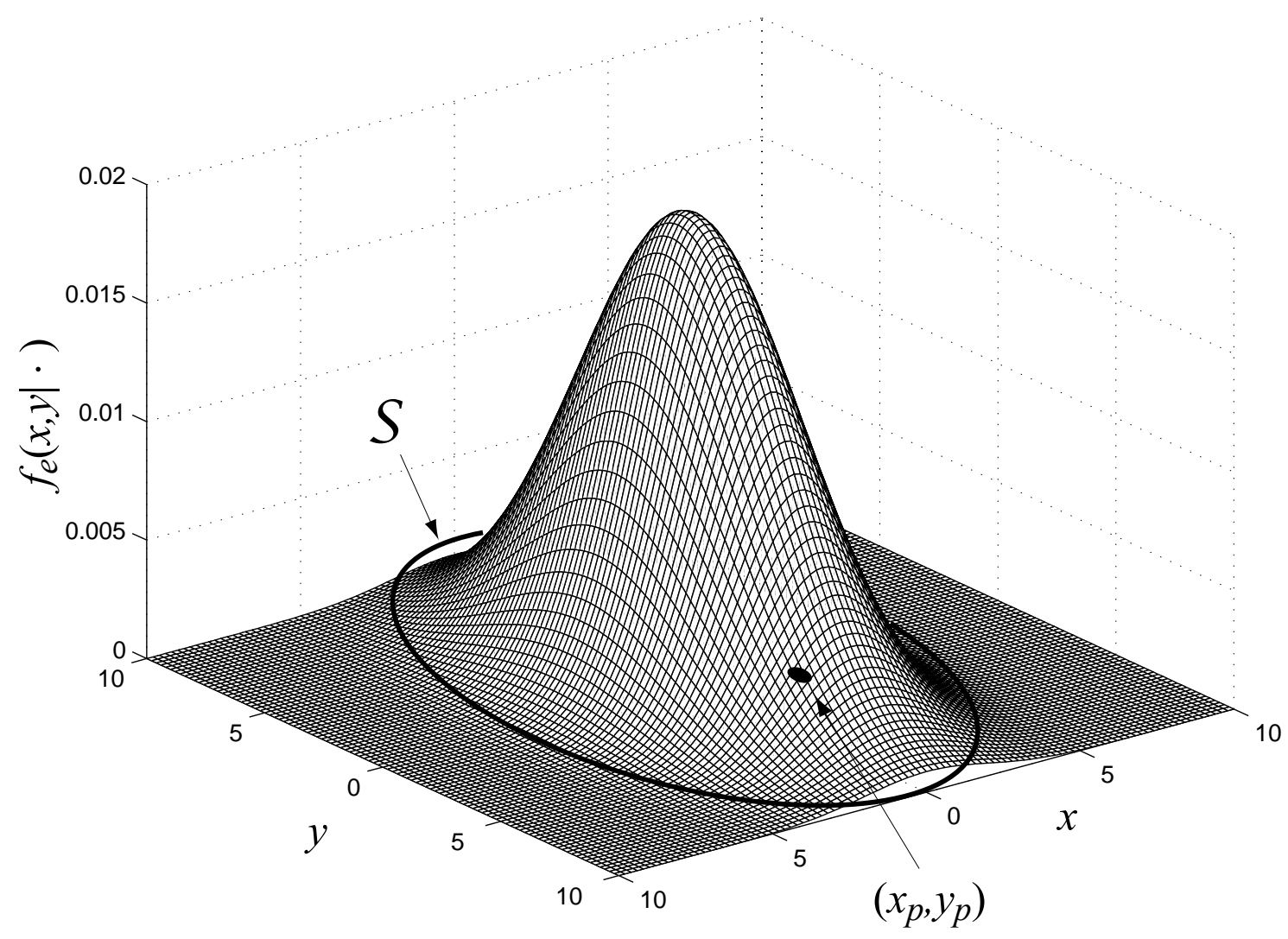

Fig. 3. A typical realization of the two dimensional Gaussian model for $f_{e}(x, y \mid \hat{x}, \hat{y}, \boldsymbol{P})$ both inside and outside elliptical region $\mathcal{S}$. $\left(x_{p}, y_{p}\right)$ is an arbitrary point inside $\mathcal{S}$. The $x, y$ scale of this plot is centered relative to an arbitrary $(\hat{x}, \hat{y})$ 


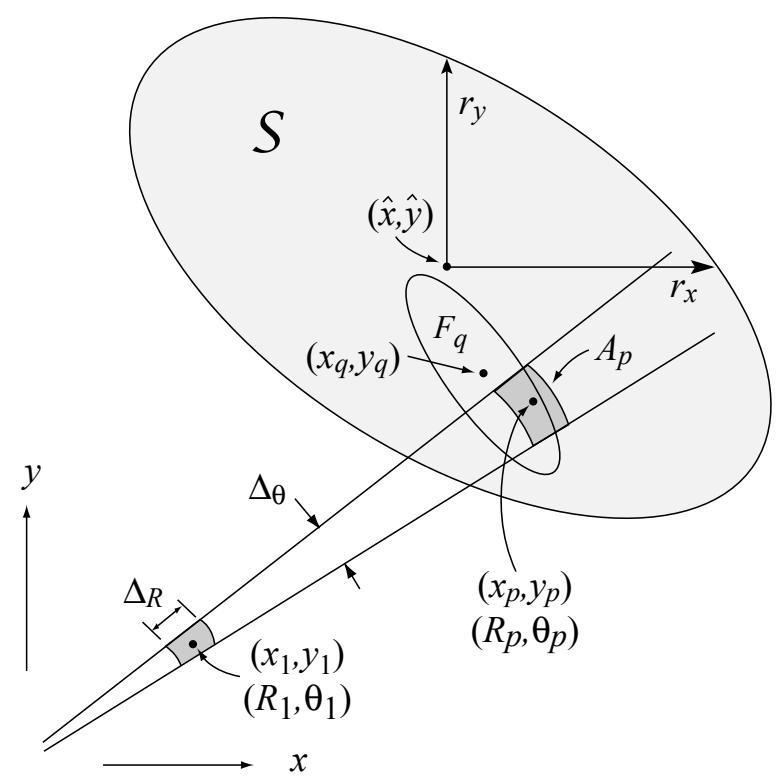

Fig. 4. Geometry of a prediction region, $\mathcal{S}$ centered on $(\hat{x}, \hat{y}) \cdot r_{x}^{2}=\beta^{2} P_{11}[k+1 \mid k]$ and $r_{y}^{2}=\beta^{2} P_{22}[k+1 \mid k]$ where $P_{i j}[k+1 \mid k]$ is the $i, j$-th matrix element in $\boldsymbol{P}[k+1 \mid k]$. Minor axis length and rotation of the ellipse are affected by off diagonal term $P_{12}[k+1 \mid k] . A_{p}$ covers the range-azimuth bin centered on $\left(x_{p}, y_{p}\right) . F_{q}$ is the echo footprint for an echo centered on some other bin, $q$. Due to uniform sampling in angle with spacing $\Delta_{\theta}$ and because $R_{p}>R_{1}$, the area of bin $A_{p}$ is greater than that of the bin centered on $\left(x_{1}, y_{1}\right)$. Note that the sizes of $A_{p}$ and $F_{q}$ relative to $\mathcal{S}$ are exaggerated to display geometry. 


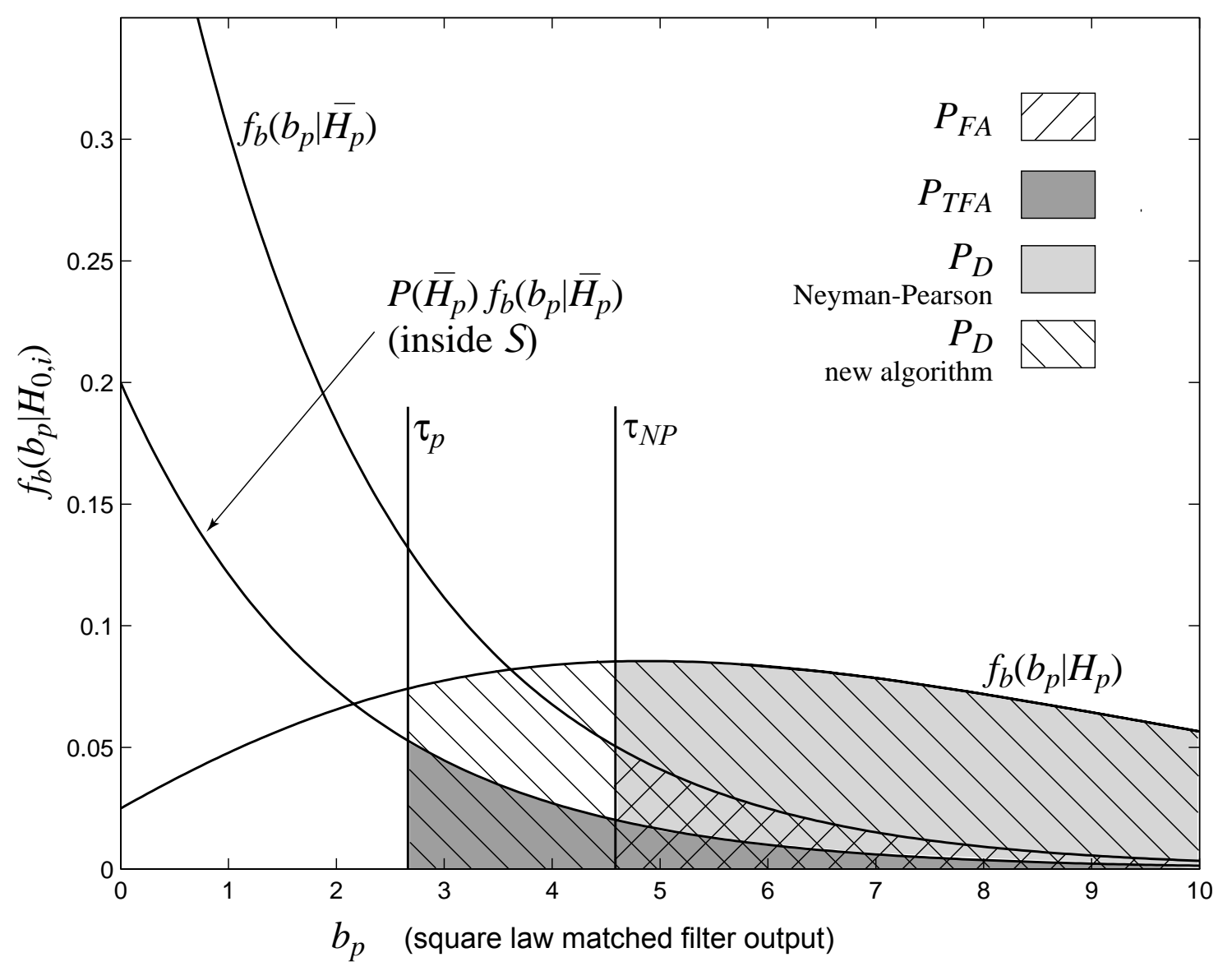

Fig. 5. Comparison of detection statistics for the N-P and proposed detectors. Inside $\mathcal{S}$ spatially varying $\tau_{p}$ is lower than conventional fixed $\tau_{\mathrm{NP}}$. This increases $P_{D}$ for the new algorithm since the integrated area beyond $\tau_{p}$ under the curve $f_{b}\left(b_{p} \mid H_{p}\right)$ is larger than the area beyond $\tau_{\mathrm{NP}}$. However $P_{T F A} \approx P_{F A}$ because $P\left(\bar{H}_{p}\right)<1$.0. For this illustration $P\left(\bar{H}_{p}\right)=0.4$ and the signal to noise ratio at the matched filter output is $4.8 \mathrm{~dB}$, which determines the non-centrality parameter $s$ for $f_{b}\left(b_{p} \mid H_{p}\right)$. 


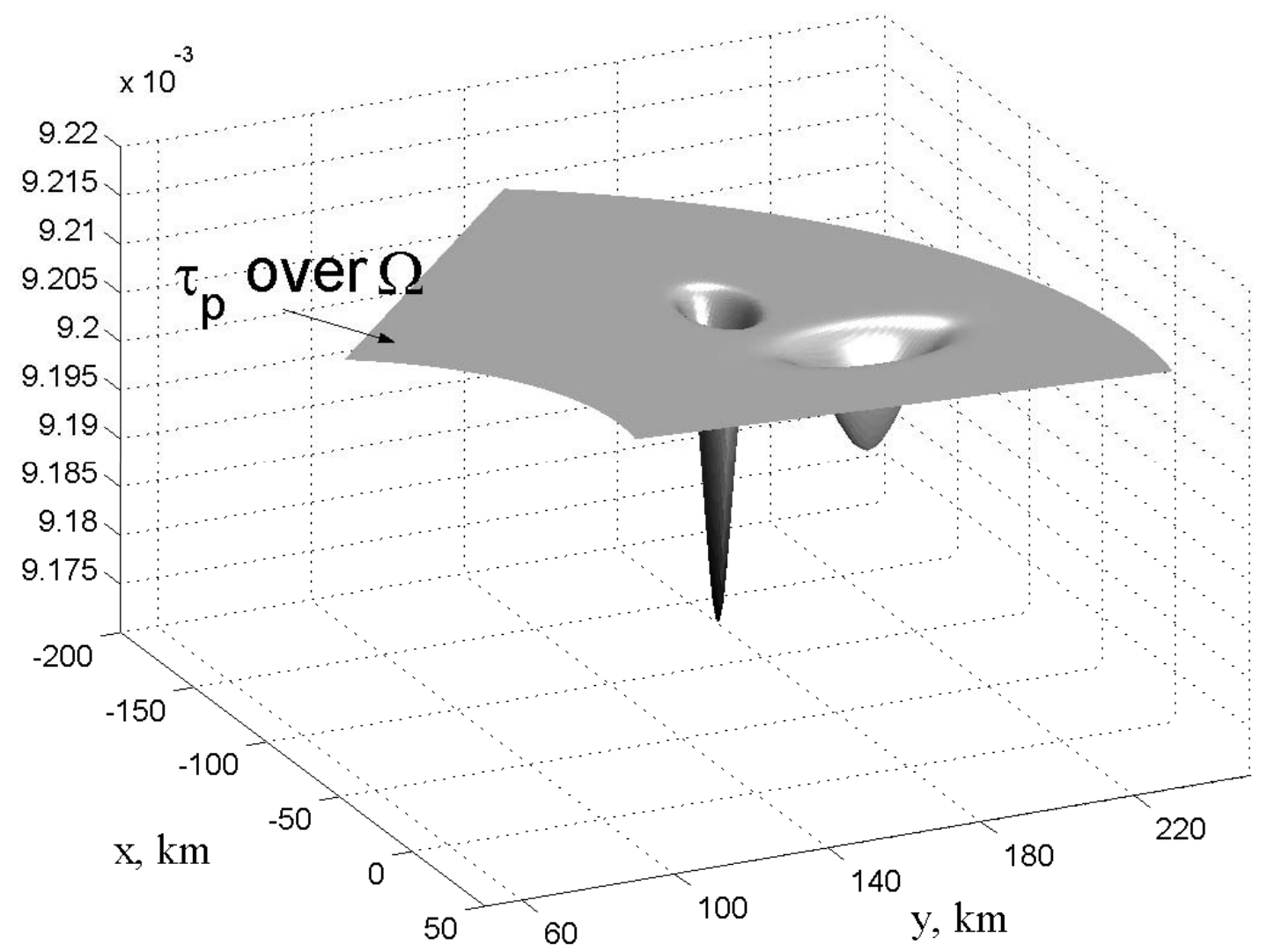

Fig. 6. The spatially varying detection threshold $\tau_{p}=\tau\left(x_{p}, y_{p}\right)$. In this example $\Omega$ contains two prediction regions $\mathcal{S}$ from two aircraft tracks. Prediction points $\left(\hat{x}_{p}, \hat{y}_{p}\right)$ correspond to the two local minima. Prior knowledge that echoes are likely over the two $\mathcal{S}$ ellipses leads to lowered threshold levels and thus the probability of detection is increased. For this plot $R_{\min }=104 \mathrm{~km}, R_{\max }=239 \mathrm{~km}, \theta_{\min }=\frac{11}{24} \pi$, $\theta_{\max }=\frac{19}{24} \pi, \sigma=0.2, P_{T F A}=1.0 \times 10^{-4}, \sigma_{\eta}^{2}=0.001, r_{x}=30 \mathrm{~km}$ for both prediction regions, and $r_{y}=15 \mathrm{~km}$ and $45 \mathrm{~km}$ respectively for the left and right regions. These parameters are typical for GBT data except that $r_{x}$ and $r_{y}$ have been increased by a factor of 10 , and $P_{T F A}$ by 100 to make detail in the plot easy to see. 


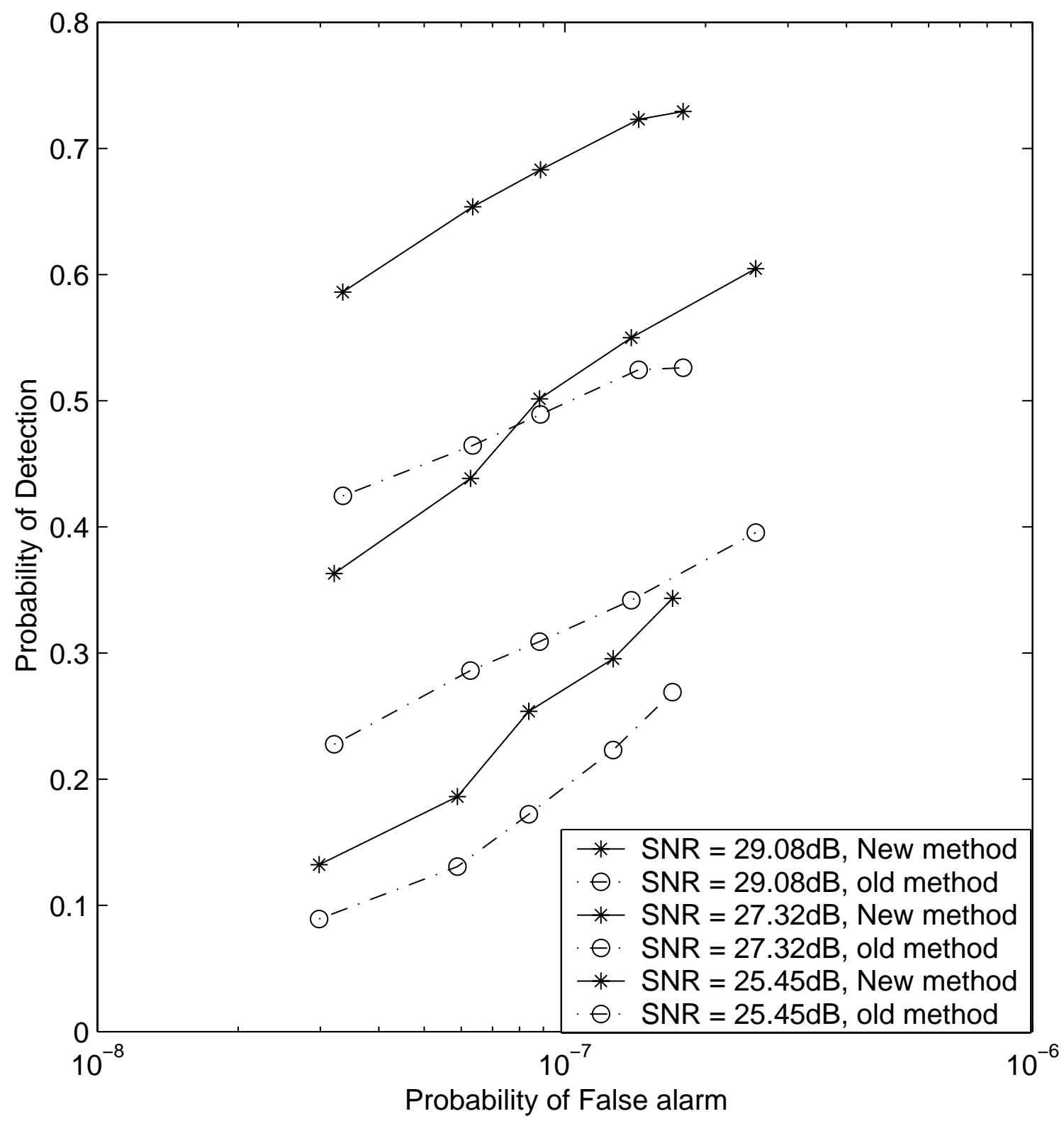

Fig. 7. Detection performance comparison for the "new" Bayesian constant $P_{T F A}$ method and "old" conventional N-P constant $P_{F A}$ approach. $P_{F A}$ vs. $P_{D}$ ROC curves are shown for a range of SNR values. $P_{F A}$ values for both algorithms are computed as the ratio of observed false alarms to the number of trials times the number of bins in $\Omega$. 


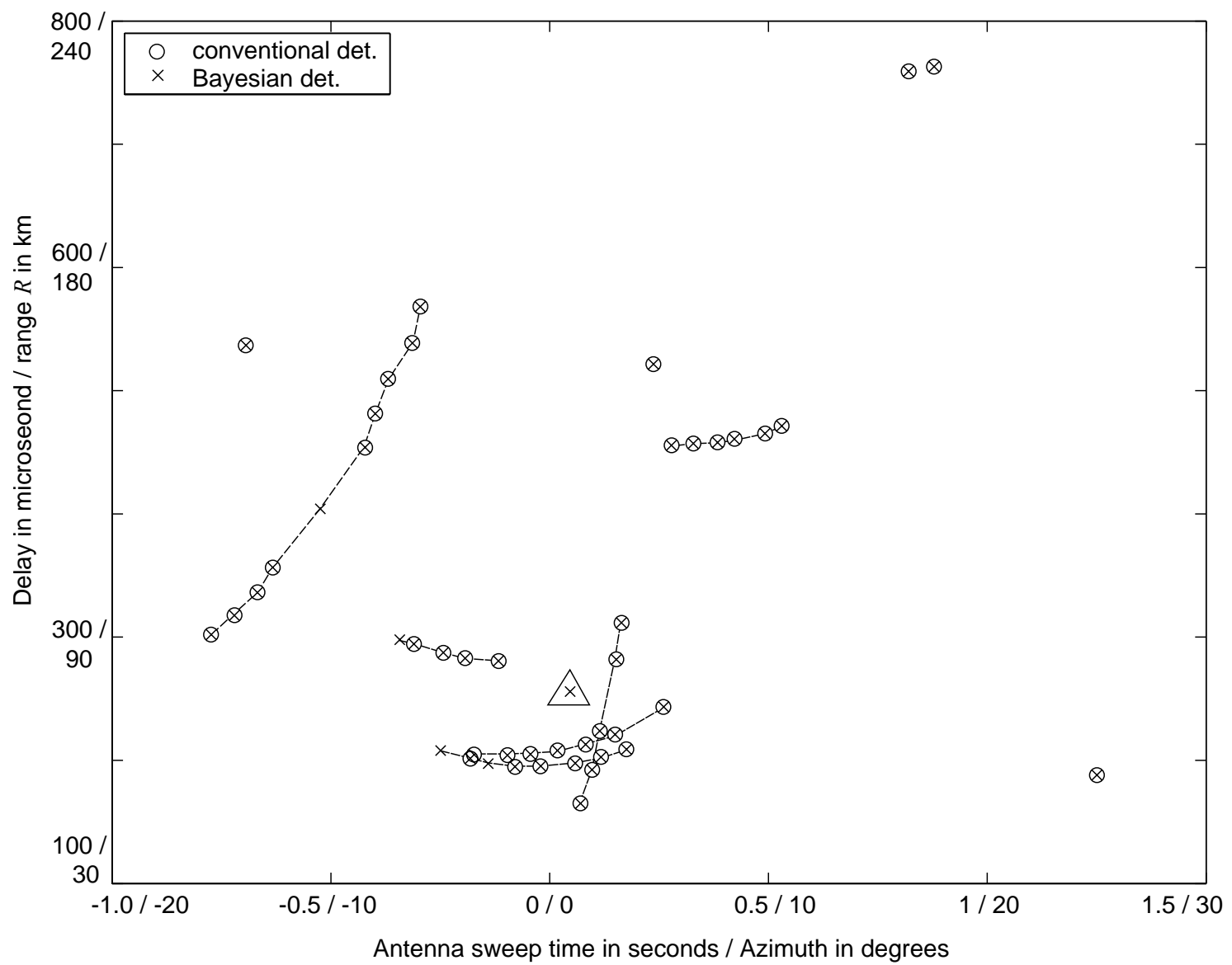

Fig. 8. All detections for the even numbered antenna sweeps in data set 2. Note that the conventional N-P detector missed four echoes (marked by crosses without circles) found by the new Bayesian method. This represents an $9 \%$ increase in finding true echoes when using Bayesian detection. The new detector added one false alarm, marked with a triangle. $P_{T F A}=P_{F A}=3.87 \times 10^{-8}$. 


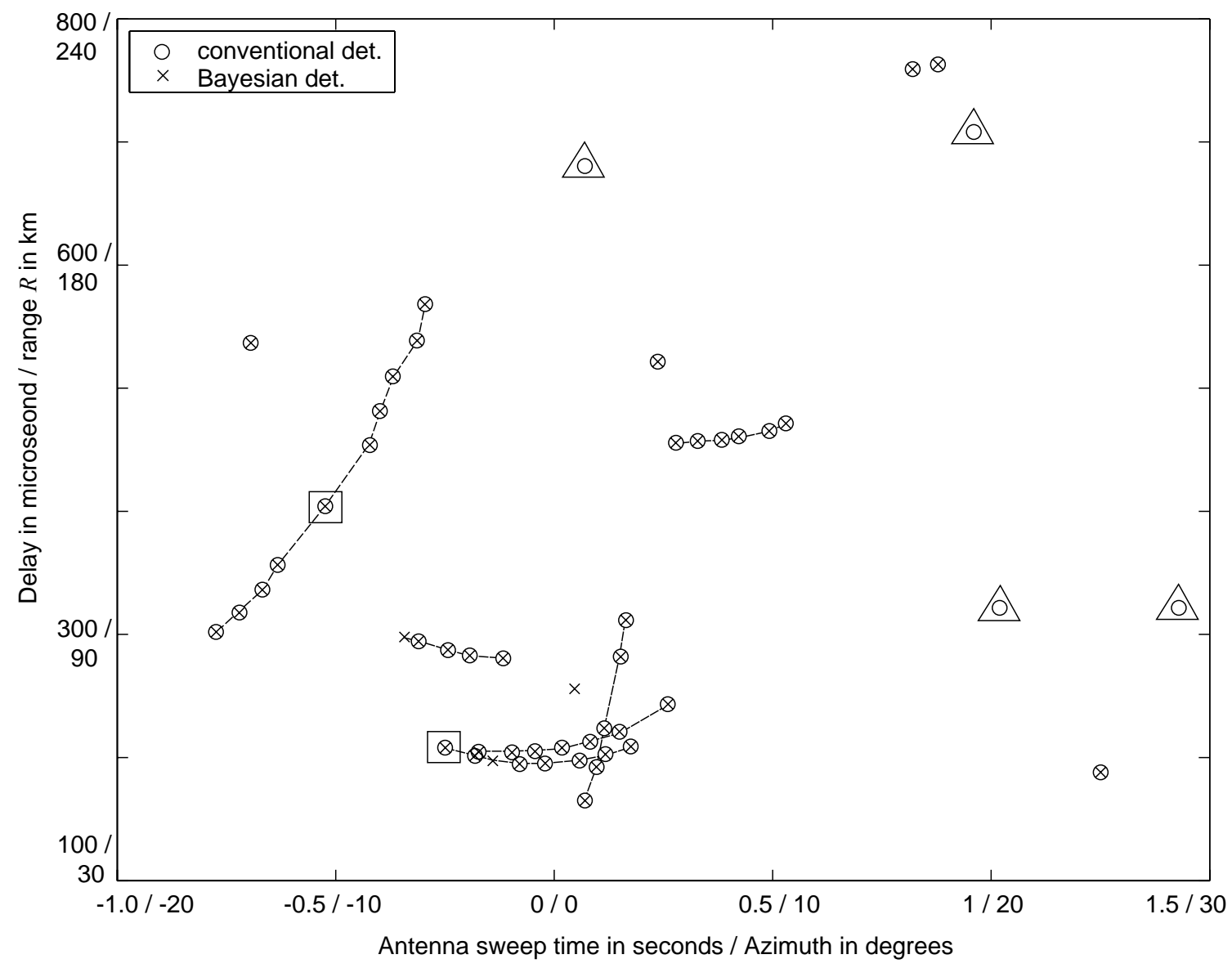

Fig. 9. Detection comparison when the N-P threshold is raised in an attempt to locate some missed echoes that were found by the Bayesian detector. Boxed detections are new correct ones. Triangles indicate new N-P false alarms. $P_{T F A}=3.87 \times 10^{-8}, P_{F A}=2.32 \times 10^{-7}$. 


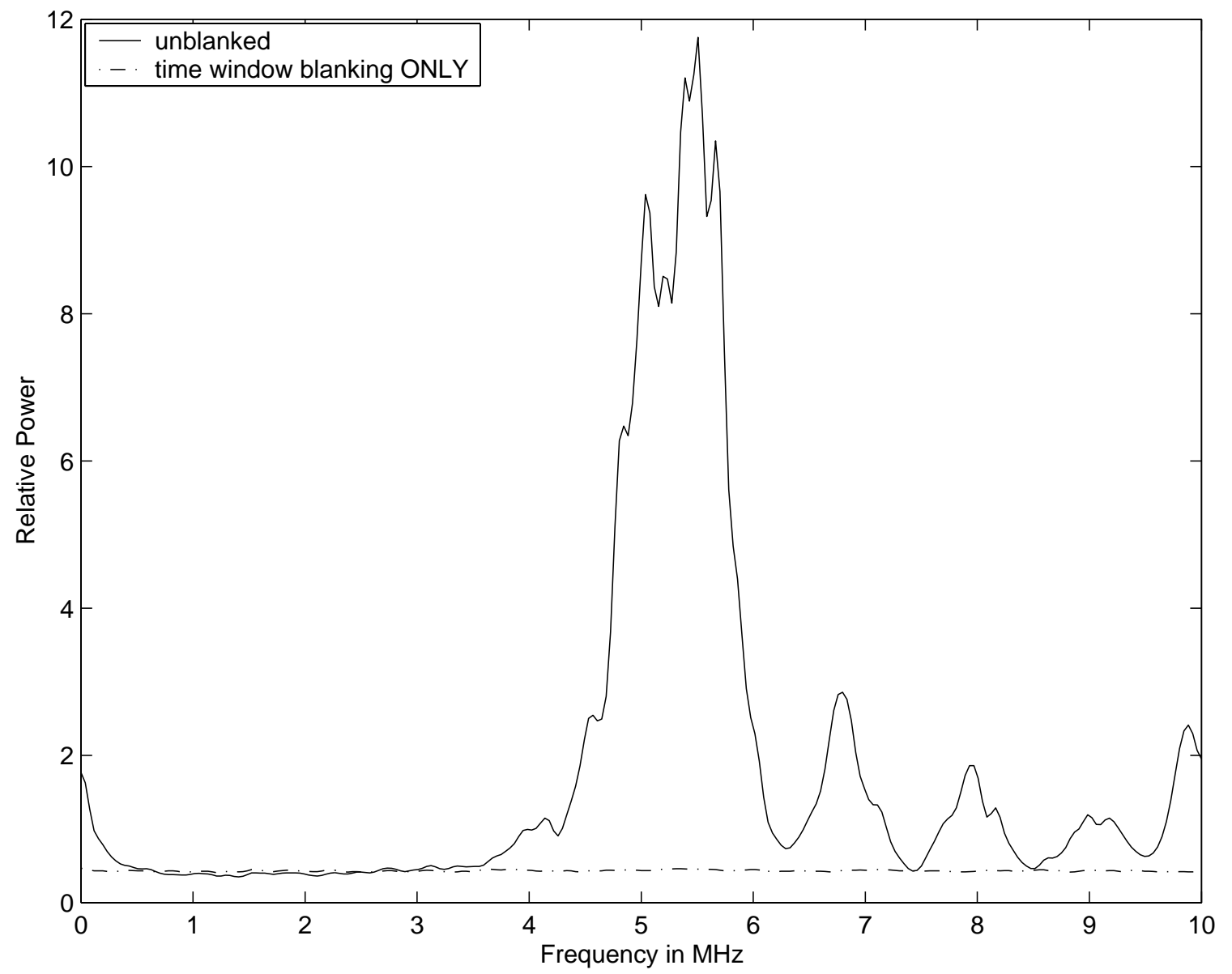

Fig. 10. Sample power spectra at the GBT showing radar RFI near 5.0 MHz. The upper curve includes no pulse blanking. The lower curve has only blanking for fixed ground echoes. 


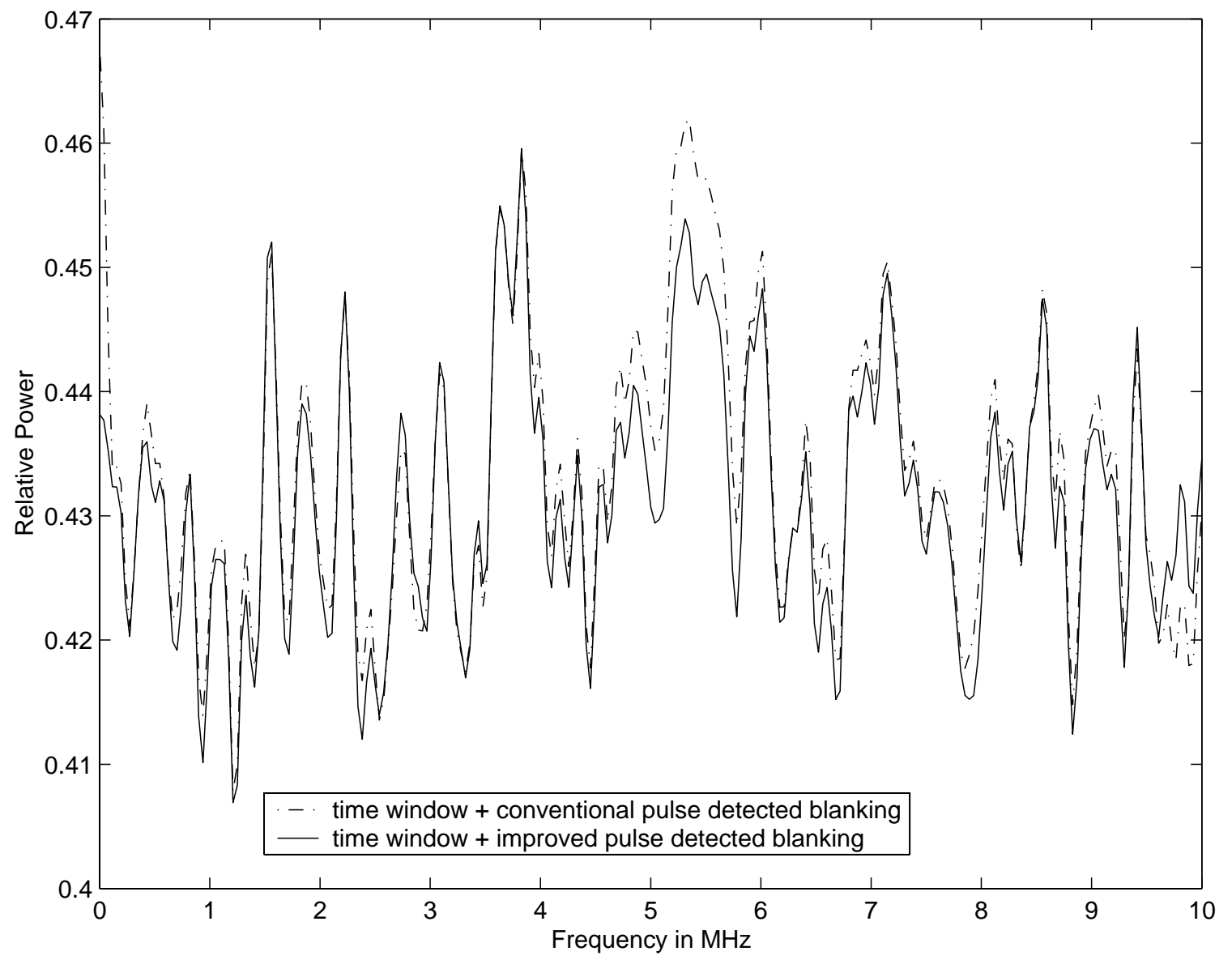

Fig. 11. Spectrum for the same 1.2 second data set seen in Figure 10, but using Kalman tracking and detected pulse blanking. Five previous antenna sweeps were used to form the track history. The upper curve resulted from conventional detection methods. The lower curve used the new Bayesian detector. Three additional aircraft were detected in this snapshot which had been missed by the conventional detector. The lower curve shows the resulting lower spectral levels in the radar RFI region near 5.0 MHz. 